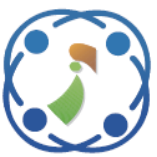

\title{
A Novel Method to Denoise Images Based on a Meta-heuristic Algorithm and Pre-learned Dictionary
}

\author{
Nouf Saeed Alotaibi ${ }^{1 *}$ \\ ${ }^{I}$ Computer Science Department, College of Science, Shaqra University, Kingdom of Saudi Arabia \\ * Corresponding author's Email: n.saeed@su.edu.sa
}

\begin{abstract}
Noise may affect images in many ways during different processes. Such as during obtaining, distribution, processing, or compressing. The Sparse Representation (SR) algorithm is one of the best strategies for noise reduction. One meta-heuristic algorithm is the Particle Swarm Optimization (PSO). This research demonstrates excellent results in noise reduction in the Fast PSO version while utilizing the SRs as well as meta-heuristic algorithms to gain. This method is known as FPSO-MP and it depends on the Pursuit Algorithm (MP) that matches. In this proposed study, a Dynamic-Multi-Swarm (DMS) method and a pre-learned dictionary (FPSO-MP) approach is presented to reduce the time for the learning dictionary calculations. The output of the denoising algorithm QPSO-MP is dependable on dictionary learning because of the dictionary size or increased number of patches. Similar to this work, a Non-locally Estimated Sparse Coefficient (NESC) is one explanation for the low efficiency of the original algorithm. Compared to the original PSO-MP method, these enhancements have achieved substantial gains in computational efficiency of approximately $92 \%$ without sacrosanct image quality. After modification, the proposed FPSO-MP technique is in contrast with the original PSO-MP method. The scientific findings demonstrate that the FPSO-MP algorithm is much more efficient and faster than the original algorithm, without affecting image quality. The proposed method follows the original technique and therefore reduces during run-time. The result of this study demonstrates that the bestdenoised images can always be accessed from the pre-learned dictionary rather than the learning dictionary developed across the noisy image during runtime. We constructed images dataset from the BSD500 collection and performed a statistical test on these images. The actual findings reveal that the suggested method is excellent for noise reduction (noise elimination) as well as highly efficient during runtime. The analytical findings indicate that both quantitative and image performance outcomes are obtained with the proposed FPSO-MP approach during its contradiction with when denoising algorithms.
\end{abstract}

Keywords: Image denoising, Sparse representation, Matching pursuit.

\section{Introduction}

Contamination of noise becomes more noticeable during processing, delivery, storing, or compression of an image. Various types of noise exist that influence the image badly. Such as Gaussian, Impulsive and Mixed noise. Denoising images is an important pre-processing phase during photograph production [1]. Image processing requires multiple pre-processing phases. The primary purpose of the denoising image is to eliminate noise from corrupted images in an attempt to approximate their original image. This is to ensure that during maintenance the edges, textures, and information of the respective features remain consistent [2]. In recent years, numerous image denoising algorithms have been proposed and successfully implemented. These algorithms have helped in increasing the accuracy of blurred images which are distorted by various kinds of noises [3, 4]. A greater description of denoising algorithms is accessible. While there are various kinds of image denoising algorithms, they are not suitable for noise reduction and run time. Primarily, three types of algorithms are utilised, namely, denoising on the domain, spatial filters as well as image denoising and image denotation. Examples of the transform domain filter image denoising 
algorithms include Wavelet, Fourier Transformations and Block-Matching and 3D filtering (BM3D) algorithms [5]. The bilateral Gaussian reference, as well as non-local mean filters, are instances of the second kind of image denouncing algorithms which heavily rely on spatial filtering. The K-Singular Value Decomposition (K-SVD) [4], Large-Scale Sparse Clustering (LSSC) [8], and Clustering-based Sparse Representation (CSR) algorithms are examples of the preceding third type of learningfocused analysis. These algorithms are designed to enhance the accuracy and performance of the images.

The denoising algorithm relies on SR to detect a correct dictionary which helps in matching the layout of the local images. The SR based denoising algorithm can be improved through dictionary efficiency with the effects of sparse coding to suit the features of the image. There are two types of the dictionary, the Static Dictionary (Wavelet, Gabor, Gabor Wavelet, Gabor Log, Log Wavelet), and the Discrete Cosine Transformation (DCT) dictionary. The second type is considered as the learning dictionary. The adaptive dictionary atoms are generated iteratively. It is also a challenging issue for the researchers to use the learning dictionary accurately. The dictionary should be extracted from the input data, which is a core concept for learning from dictionaries. The new sparse dictionary learning strategies are promoted because the image transmission usually requires to represent the input data utilizing as little as possible. Pre-determined dictionaries, such as Fourier or wavelet Transformations had to be included in the general procedure previous to that technique.

The Processor requires few minutes or more to learn from the dictionary for a standard issue. The implementation of the K-SVD method is, therefore, overloaded in large-scale images. The usage of the learning dictionary in the denoising algorithm remains a challenging problem. Researchers have encountered problems when it comes to identifying an appropriate image denoising strategy. SR method is most widely used for images representing Gaussian noise. The K-SVD method implements two subproblems, Sparse Coding, and Dictionary Updating. Nearly all sub-problems in [4] rely on heuristic sampling techniques. OMP achieves sparse coding under its over-complete dictionary as well as the dictionary is updated and calculated using columnwise sequential SVD. The accurate use of the learning dictionary is still a challenging issue when using it with the denoising method. Researchers are facing a challenge in seeking an appropriate imaging technique denoising.
The goal of this study is to reduce the adaptive dictionary's computational complexity and improve test image denoising quality. This paper includes MP as well as a dictionary learned from the latest quick PSO algorithm. This study has enhanced the PSO to reduce the difficulty of the calculation as well as the time of execution.

Two important performance measures for denoising evaluation indicators are noise reduction and numerical efficiency. In other words, most denoising apps require an agreement between noise reduction as well as computational expense. This study has explored the usefulness of the computational difficulty in a learning dictionary which is one of the long-term modules of the original PSO-MP algorithm. Experimental findings suggest that our fast PSO-MP (FPSO-MP) process, with two technological developments, has generated highly competitive output in terms of noise reductions, computational complexity and denoising algorithm as compared to its simple execution. These enhancements relate significant advantages of up to 92\% computational efficiency without compromising on image quality compared to the original PSO-MP method. After modification, the proposed FPSO-MP technique is contrasting to the original PSO-MP method. The scientific findings show that our proposed FPSO-MP algorithm is much more efficient as well as faster than the original algorithm, without having to sacrifice image quality.

The rest of this paper is arranged as follows. The new FPSO-MP approach is addressed in section 2 along with two improvements in the initial PSO algorithm. Section 3 has performed checks and analysis based on the experimental outcomes and performance of the proposed FPSO-MP algorithm and the other denoising algorithms. In section 4, this study is concluded.

\section{The proposed method}

\subsection{Matching pursuit (MP)}

MP is a wasteful algorithm, which uses the decomposition of the signal depending on an atom which is the part of the redundant dictionary. While MP is added, the appropriate atom may be identified from just the dictionary atoms, which could be accessible in each iteration. Let $D=\left\{\mathrm{g}_{\gamma}\right\}_{\gamma \in \Gamma}$ is dictionary atoms, with an undefined scale image of size $\mathrm{z} \times c$. Here, $\Gamma$ is the set of all indexes $\gamma$ and $\left\|g_{\gamma}\right\|=1$ [1]. Considering $y$ giving out a random image. An MP's first step is to estimate $x$ by projecting it on a vector $g_{\gamma_{0}} \in D$. 


$$
x=<x, g_{\gamma_{0}}>g_{\gamma_{0}}+R e_{x}
$$

Where, $<x, g_{\gamma_{0}}>g_{\gamma_{0}}$ is the atom $g_{\gamma_{0}} y$ projection, $R e_{x}$ is the initial signal residual, and $g_{\gamma_{0}}$ is orthogonal to the residual $R_{x}$ [1]:

$$
\begin{aligned}
\|x\|^{2} & =\left|<x, g_{\gamma_{0}}>\right|^{2}\left\|g_{\gamma_{0}}\right\|^{2}+\left\|R e_{x}\right\|^{2} \\
\|x\|^{2} & =\left|<x, g_{\gamma_{0}}>\right|^{2} \\
& +\left\|R e_{x}\right\|^{2}, \text { where }\left\|g_{\gamma_{0}}\right\|^{2}=1
\end{aligned}
$$

The equation $\left\|R e_{x}\right\|^{2}=\|x\|^{2}-\left|<x, g_{\gamma_{0}}>\right|^{2}$ has to minimize. The equation $g_{\gamma_{0}} \in D$ is required to be selected to maximize $\left|<x, g_{\gamma_{0}}>\right|$. The original signal can be rebuilt using the selected $g_{\gamma_{n}}$ atom using the following $Q$ iteration:

$$
x \approx \sum_{q=0}^{Q-1}<R e_{x}^{q}, g_{\gamma_{q}}>g_{\gamma_{q}}
$$

Several of the key stages in denoising systems are the method of producing $g_{\gamma}$. The Gaussian method is often used in Eq. (5). The basic function is a Gaussian which is a one-directional function and the second factor in the Gaussian dimension. A Gaussian function is being used in the TLS model to construct the pre-learned dictionary. The corresponding formula is going to build the Gaussian method.

$$
g_{\gamma}=\left(2-4 u^{2}\right) e^{-\frac{1}{4}\left(u^{2}+m^{2}\right)}
$$

In Eq. (5), the development of $x$ and $y$ directions for the TLS rotation dictionary is used, i.e. (translation and libration in $x, y$ directions $T[L]_{x}, T[L]_{y}, L_{x}, L_{y}$ and screw rotation $S_{[L]}$ ) respectively. Where, $u$, and $m$ are the number of rows and vectors of the pre-learned dictionary which uses the TLS pattern to estimate $u, m$.

$$
\begin{aligned}
& u=\frac{\left(T[L]_{x} \times \cos \left(S_{[L]}\right)+T[L]_{y} \times \sin \left(S_{[L]}\right)\right)}{2^{\left(\frac{s x}{N N}\right)}} \\
& m=\frac{\left(T[L]_{y} \times \cos \left(S_{[L]}\right)-T[L]_{x} \times \sin \left(S_{[L]}\right)\right)}{2^{\left(\frac{s t y}{N N}\right)}}
\end{aligned}
$$

Where $S_{[L]}, s t x, T[L]_{x}, T[L]_{y}$, and sty is a, screw rotation angle, $x, y$ are the translation in axis and random population columns. The total amount of TLS model variables is $5, N N=5$.

\subsection{Particle swarm optimization (PSO)}

PSO is implemented in [6], by Kennedy and Eberhart, emulates bird flocking attitudes to fix optimization issues. In PSO, any solution is considered to be a particle. All particles have velocities and fitness values. By knowing from the previous knowledge of all the particles, we get to know that the particles travel across the $D$ dimensional problem space. Moreover, the particles continue to travel throughout the searching phase into a better search location. The velocity vel $_{i}^{d}$ and location pos ${ }_{i}^{d}$ changes for the $i^{\text {th }}$ particle's $d^{\text {th }}$ dimension:

$$
\begin{aligned}
& \text { vel }_{i}^{d}=\beta \times \text { vel }_{i}^{d}+a_{1} \times \text { random } 1_{i}^{d} \\
& \times\left(\text { pbest }_{i}^{d}-\operatorname{pos}_{i}^{d}\right)+a_{2} \times \operatorname{random} 2_{i}^{d} \\
& \times\left(\text { gbest }^{d}-\operatorname{pos}_{i}^{d}\right) \\
& \quad \operatorname{pos}_{i}^{d}=\operatorname{pos}_{i}^{d}+\text { vel }_{i}^{d}
\end{aligned}
$$

Although $a_{1}$ and $a_{2}$ are the momentum constants, random $1_{i}^{d}$ and random $_{i}^{d}$ are the two standard distributed random numbers within the range $[0,1]$. $\operatorname{pos}_{i}=\left(\operatorname{pos}_{i}^{1}, \operatorname{pos}_{i}^{2}, \operatorname{pos}_{i}^{3}, \ldots, \operatorname{pos}_{i}^{D}\right)$, pbest $_{i}=$ (pbest ${ }_{i}^{1}$, pbest $_{i}^{2}$, pbest $_{i}^{3}, \ldots$, pbest $\left._{i}^{D}\right)$

and gbest $=\left(\right.$ gbest $^{1}$ gbest $^{2}$, gbest $^{3}, \ldots$, gbest $\left.^{D}\right)$ are the locations of the particle $i^{t h}$ dimension, the strongest prior location for the pbest $_{i}$ fitness cost of the $i^{\text {th }}$ particle and the strongest discovered location for the whole population.

\subsection{Fixed or pre-learned dictionary}

The dictionary is the replication of the initial algorithm for any outer loop iteration. The denoising or medium noisy image is collected to train patches. These training patches are clustered through different groups and PCA sub-dictionary which is acquired by each cluster. A lightweight sub-dictionary of PCA is selected to code it for a particular patch. Since dictionary construction from image patches occurs at runtime, therefore, this would be relatively higher time difficulty, especially for large image size. In reality, certain consideration must be fulfilled to generate the dictionary that can effectively portray the quality of the image: (1) training the dictionary itself on the noisy image (produced at runtime as well as incrementally modified, earlier known as the learned dictionary, obtained in the initial algorithm) and (2) training the dictionary immediately on a highquality image rather than a blurry one. In this 


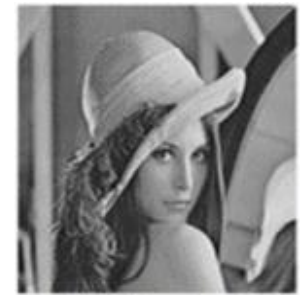

(a)

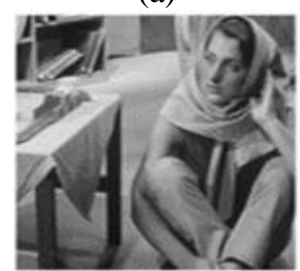

(d)

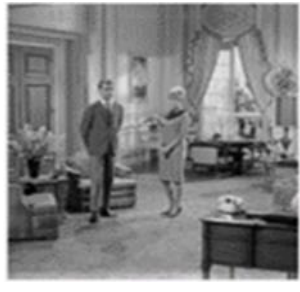

(b)

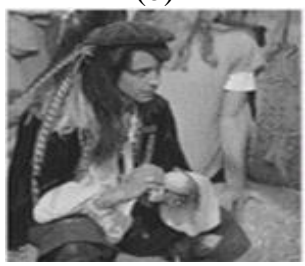

(e)

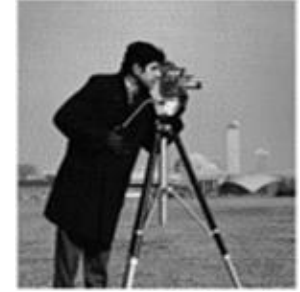

(c)

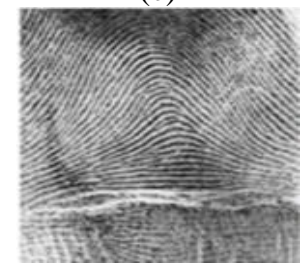

(f)

Figure. 1 Six test images. :(a) img1, (b) img2, (c) img3, (d) img4, (e) img5, and (f) img6. The img1, img2, and img3 are $256 \times 256$. All other images are $512 \times 512$.

research, instead of an optimized learned dictionary runtime, the pre-learned dictionary has been used, which has greatly reduced the run time. Every internal iteration of the initial algorithm includes the following. The decomposition of the blurry image into inconsistent patches, the sparse coding of image patches, the calculation of sparse coefficients, and the reconstruction of the noisy image from the estimated patches [2, 3]. In such modules, the dictionary operates a significant part. As we know that it is not feasible to reach the initial image equal to the noisy one. Therefore, when implementing the initial algorithm to the denoise image, the dictionary is going to produce another smooth image, in which its elements vary from the components of a noisy image. The comparison images representing the components of the sample images in Figs. 1 and 2 distinctively. This can also be used to calculate the reliability of the pre-learned dictionary technique. The denoising effects of the original method are not specific to the sample images used for the advancement of the dictionary.

A pre-learned dictionary also being used to improve the PSO-MP algorithm.

\subsection{DMS of population}

A current version of the PSO algorithm is the focus of the DMS-PSO strategy. The difference between PSO and DMS-PSO is that the swarms are both large and smaller [8]. The DMS-PSO algorithm is a community topology with two main features. Also, the DMS defines the total PSO algorithmpopulation into smaller swarms. Such swarms are constantly re-clustered using different proposals for grouping and exchanging of all this data among them. Fig. 3. shows the DMS-PSO strategy search [1].

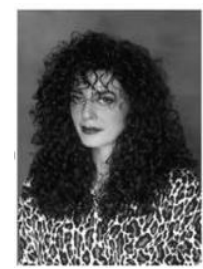

(a)

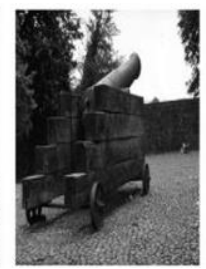

(b)

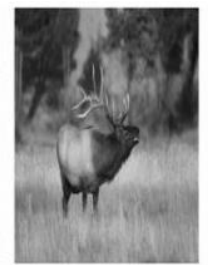

(c)

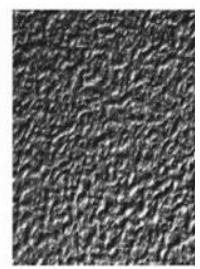

(d)
Figure. 2 Additional images were collected randomly from numerous image databases BSD500 [7]

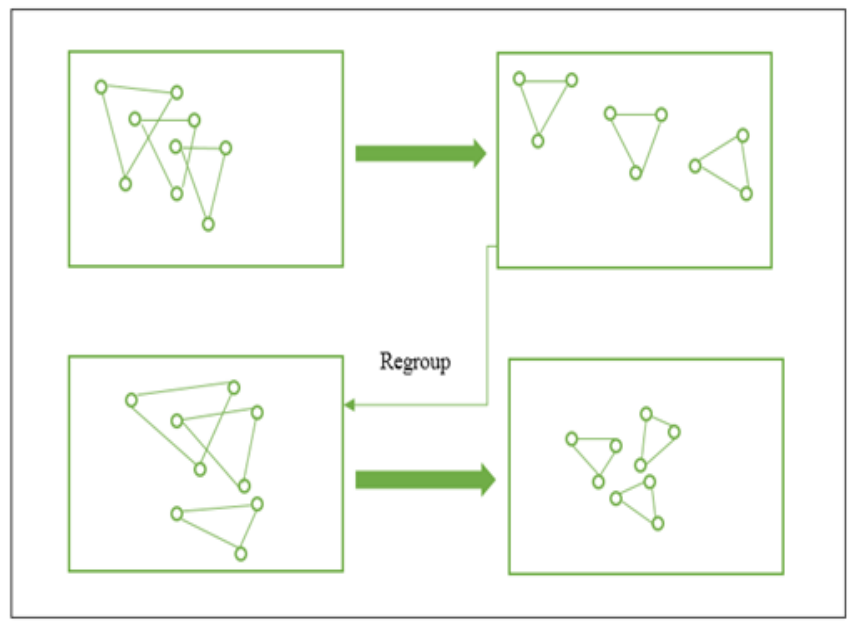

Figure. 3 DMS-PSO strategy search [1]

\subsection{The proposed (FPSO-MP) method}

The first enhancement in our proposed method is to construct the pre-learned dictionary from highquality textured images instead of the overcompleted dictionary generated. The generated dictionary is created from its own from the defined noisy image or the inadequately denoised image. The second proposed enhancement is to use the DMS over the algorithm of the PSO (DMS-PSO). The population is broken into tiny parts while utilizing DMS. That group is going to be utilizing their 


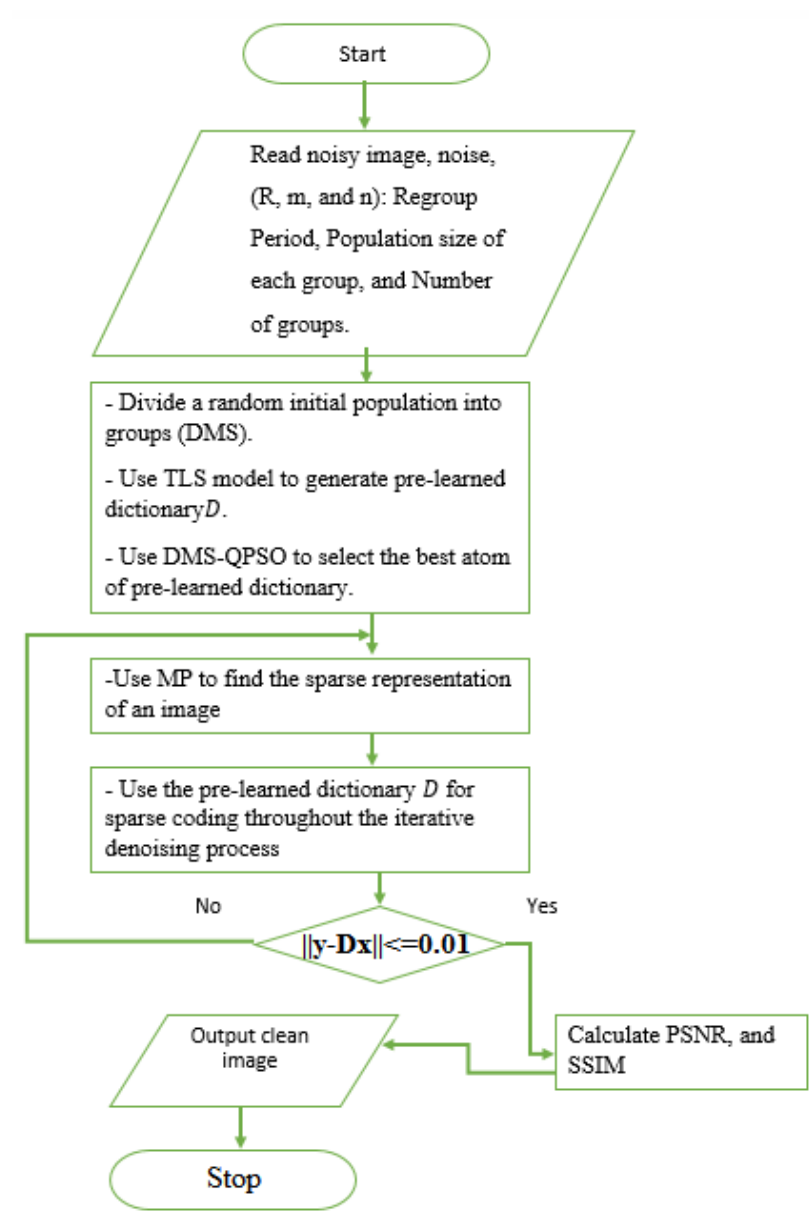

Figure. 4 The flow chart of the proposed method

participants to discuss the space. There is going to be no drop in the complexity of an entire population during this process because the knowledge produced by each swarm would be swapped. The leaders of each swarm group would be normally distributed in a generation (i.e. the period of regrouping). Fig. 4 demonstrates the main steps of the proposed (PSOMP) method.

\section{The numerical results}

\subsection{Comparison of the proposed algorithm with the original version}

We have performed our testing on the proposed FPSO-MP approach on the BSD500 [7]. We constructed a dataset of 200 images from the BSD500 collection and implemented a statistical test on these images. The comparison images consist of several styles of scenes such as indoor, outdoor and portrait scenes. The computational complexity of the proposed and initial algorithms is measured. The testing is performed on a SAMSUNG PC, running Windows 10 with i7 CPU, MATLAB R2013 64-bit, and 8 GB RAM.

\subsubsection{Complexity analysis}

An initial method of main computing costs is described as follows. The quality of the denoising initial method is heavily relied on dictionary learning, especially when it increases the dictionary size or the number of training patches. Previously, one of the given explanations for the low performance of the initial algorithm of a Non-local Approximate Sparse Coefficient (NESC) was relying on a corresponding patch. This patching process is a time-consuming procedure that is fairly close to the initial algorithm used for diagnosis. The isotropic atom used throughout the initial algorithm is to construct the dictionary, which is a time-consuming process and this also explains the poor performance of the initial algorithm. While the original algorithm is useful to minimize noise, the measurement expense of these difficulties is limited in large-scale images. Additionally, the testing for the initial method process is not sensitive to the dictionary sample images material. Moreover, the pre-learned dictionary is being used to speed up the initial algorithm. The original denoising algorithm requires the complexity of $O\left(P I^{2}\right)$, where $P$ is the patch pixel quantity, and $I$ is the image pixel quantity. Moreover, the time required to denoise a single $512 \times 512$ image is too long. The duration for an outer loop is roughly $472 \mathrm{~s}$ with unoptimized MATLAB codes on a PC fitted with Intel Core (TM) i7 CPU and $8 \mathrm{~GB}$ RAM for $512 \times 512$ images. In a simple outer loop, the computation of dictionary learning, as well as weighting, requires about $82 \mathrm{~s}$ and $390 \mathrm{~s}$, which requires about $1032 \mathrm{~s}$ to denoise a $512 \times 512$ image. Both improvements (DMS population as well as prelearned dictionary) were used to improve the computational efficiency of the initial algorithm to minimize the processing time without muchsacrificing on the image quality. The pre-learned dictionary function was constructed successfully in reducing the proposed algorithm's running time.

The pre-learned dictionary applied immediately on our proposed method (it doesn't require long to set up) while it requires more than $82 \mathrm{~s}$ for image processing to operate efficiently. This means that the computational complexity of the proposed method is much smaller than the local version of the proposed algorithm. Fig. 6 demonstrates the average quality of the suggested algorithm, which exceeds the quality of the algorithm suggested. Fig. 7 Shows the average implementation time of the suggested method, which is below the equivalent value of the initial method. This indicates the potential benefits of our proposed method. 


\subsubsection{Noise elimination}

The proposed and initial methods are represented by a PSNR meaning of denoised images in Table 2. It is recommended that the proposed approach should be used with broad images because of its limited runtime and acceptable performance of denoised images. The average values of PSNR for the proposed method is greater than those for the proposed algorithm. The key reason for this is the population separated into different classes. Table 3 demonstrates the comparison time of execution (in seconds) of the proposed and initial method under varying noise levels from 5 to 90 . The proposed method has faster execution than the initial method. In the case of visible analysis, the findings of both methods are quite similar. This implies that the new method performs well and the initial method is efficient in reducing the run time. The variations between both the findings denoised denounced can be seen in Fig. 5. The proposed and initial method show a similar sigma and image. The proposed method follows the original method and therefore successfully manages to reduce the run time. The testing and analysis of this study show that the bestdenoised images are accessible from the pre-learned dictionary rather than the learning dictionary developed across the noisy image during runtime. Natural images successfully match with the prelearned dictionary. In short, the actual findings reveal that the suggested method is excellent for noise elimination and running time. Table 1 displays the

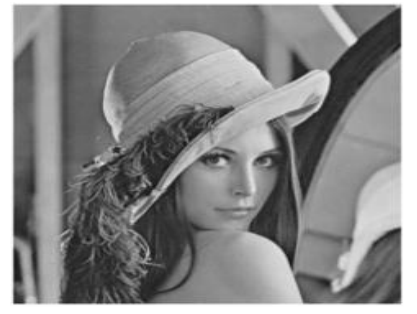

(a)

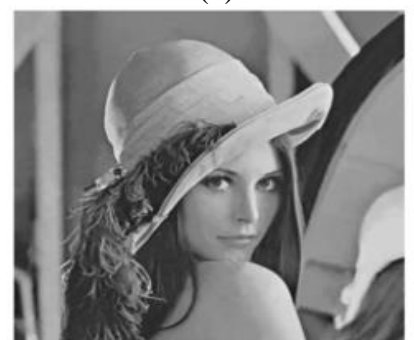

(c)

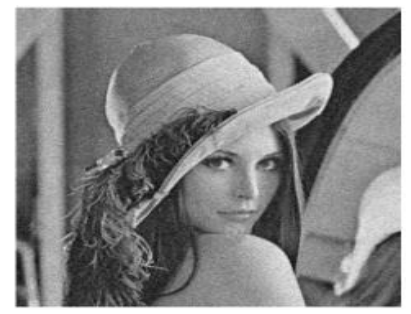

(b)

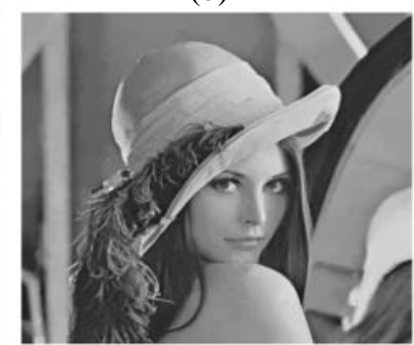

(d)
Figure. 5 Denoised findings of a low noise on img.1 ( $\sigma=$ 25 ) using original (initial) and proposed, respectively. (a) Original image; (b) corrupted image; (c) denoised image using the initial method, and (d) denoised image using the proposed method

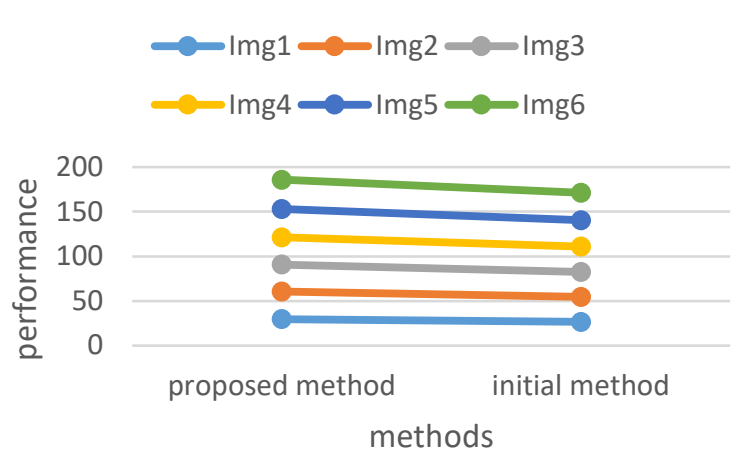

Figure. 6 Displays average PSNR performance for the suggested and initial denoising methods on all sample images
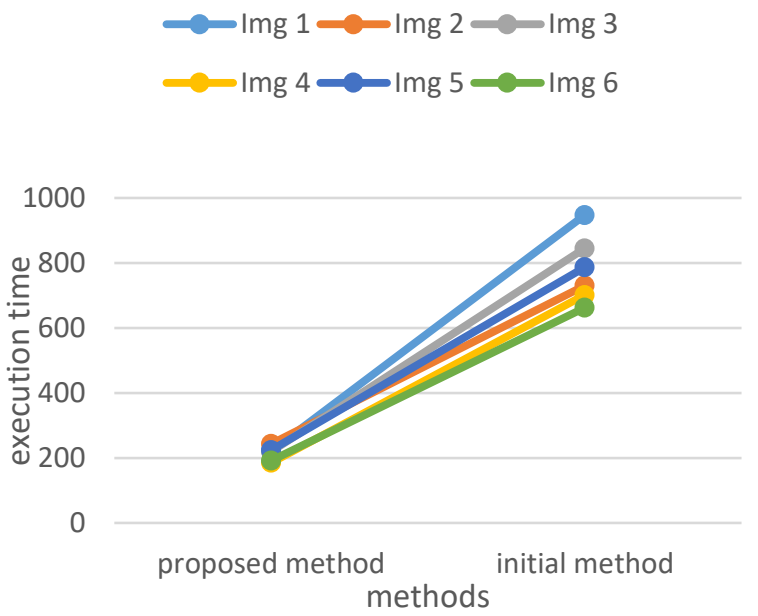

Figure. 7 Average execution time for the suggested and initial denoising method on all sample images

pre-learned dictionary that was equipped to recognize the meaning of the images obtained on certain excellent quality images. Table 1 shows that the PSNR findings are higher than 133 decibels (dB), indicating that the accuracy of the reconstructed image is acceptable.

\subsection{Comparison between the proposed and the state-of-the-art algorithms}

The denoising efficiency of the proposed method is equivalent to the state-of-the-art denoising techniques, 1) BM3D [5], 2) LSSC [9], 3) edgepreserving image denoising (EPID) (Gijbels, Lambert and Qiu, 2006), 4) WNNM [11], 5) SNLM $[12], 6)$ BM3D-SAPCA [13], 7) FastNLM (Seynnes and Cronin, 2020), and 8) FNCSR [15]. To make a fair distinction of both the suggested method and the state-of-the-art algorithms, the images evaluated were the same, providing the very same size as well 
Table 2. Displays PSNR (in decibel) compared of the suggested method with the initial method under various noise rates between 5 as well as 90. The bold values suggest full PSNR values

\begin{tabular}{ccccccccccc}
\hline \multirow{2}{*}{ Images } & \multicolumn{2}{c}{$\sigma=5$} & \multicolumn{2}{c}{$\sigma=25$} & \multicolumn{2}{c}{$\sigma=45$} & \multicolumn{2}{c}{$\sigma=75$} & \multicolumn{2}{c}{$\sigma=90$} \\
\cline { 2 - 10 } Img1 & suggested & initial & suggested & initial & suggested & initial & suggested & initial & suggested & initial \\
\cline { 2 - 10 } Img2 & $\mathbf{3 8 . 4}$ & 35.3 & $\mathbf{3 3 . 8}$ & 31.6 & $\mathbf{2 7 . 5}$ & 24.9 & $\mathbf{2 5 . 8}$ & 21.6 & $\mathbf{2 2 . 3}$ & 19.8 \\
Img3 & $\mathbf{3 5 . 8}$ & 35.7 & $\mathbf{3 3 . 9}$ & 30.2 & $\mathbf{3 0 . 7}$ & 28.5 & $\mathbf{2 8 . 1}$ & 24.2 & $\mathbf{2 4 . 9}$ & 21.3 \\
Img4 & $\mathbf{3 6 . 4}$ & 34.2 & $\mathbf{3 1 . 4}$ & 29.2 & $\mathbf{3 0 . 6}$ & 27.8 & $\mathbf{2 7 . 2}$ & 25.4 & $\mathbf{2 5 . 7}$ & 23.6 \\
Img5 & $\mathbf{3 7 . 5}$ & 35.6 & $\mathbf{3 4 . 5}$ & 31.3 & $\mathbf{3 0 . 8}$ & 28.4 & $\mathbf{2 7 . 3}$ & 24.7 & $\mathbf{2 4 . 4}$ & 23.5 \\
Img6 & $\mathbf{3 9 . 3}$ & 38.2 & $\mathbf{3 6 . 1}$ & 34.3 & $\mathbf{3 1 . 3}$ & 27.4 & $\mathbf{2 9 . 1}$ & 27.6 & $\mathbf{2 6 . 3}$ & 24.3 \\
Average & $\mathbf{3 7 . 5}$ & 35.4 & $\mathbf{3 4 . 0}$ & 31.6 & $\mathbf{3 3 . 4}$ & 31.3 & $\mathbf{3 0 . 4}$ & 27.3 & $\mathbf{2 5 . 1}$ & 22.5 \\
\hline
\end{tabular}

Table 3. Demonstrates comparison time of execution (in seconds) of the proposed and initial method under varying noise levels from 5 to 90 . The bold values show the lowest period to implement

\begin{tabular}{ccccccccccc}
\hline \multirow{2}{*}{ Images } & \multicolumn{2}{c}{$\sigma=5$} & \multicolumn{2}{c}{$\sigma=25$} & \multicolumn{2}{c}{$\sigma=45$} & \multicolumn{2}{c}{$\sigma=75$} & \multicolumn{2}{c}{$\sigma=90$} \\
\cline { 2 - 10 } & suggested & initial & suggested & initial & suggested & initial & suggested & initial & suggested & initial \\
\cline { 2 - 10 } Img1 & $\mathbf{2 6 3}$ & 1032 & $\mathbf{2 0 4}$ & 1009 & $\mathbf{2 4 2}$ & 935 & $\mathbf{1 9 4}$ & 923 & $\mathbf{2 0 3}$ & 837 \\
Img2 & $\mathbf{1 7 5}$ & 783 & $\mathbf{2 9 2}$ & 837 & $\mathbf{3 6 4}$ & 683 & $\mathbf{2 0 3}$ & 763 & $\mathbf{1 8 2}$ & 585 \\
Img3 & $\mathbf{1 6 9}$ & 981 & $\mathbf{2 8 4}$ & 1047 & $\mathbf{2 7 3}$ & 794 & $\mathbf{1 9 8}$ & 672 & $\mathbf{1 7 3}$ & 731 \\
Img4 & $\mathbf{2 6 0}$ & 692 & $\mathbf{1 2 9}$ & 629 & $\mathbf{2 5 1}$ & 842 & $\mathbf{1 7 3}$ & 753 & $\mathbf{1 1 2}$ & 592 \\
Img5 & $\mathbf{2 1 2}$ & 719 & $\mathbf{1 1 8}$ & 768 & $\mathbf{3 7 2}$ & 927 & $\mathbf{2 1 7}$ & 683 & $\mathbf{2 0 1}$ & 836 \\
Img6 & $\mathbf{2 0 5}$ & 659 & $\mathbf{1 5 3}$ & 593 & $\mathbf{1 3 7}$ & 494 & $\mathbf{2 8 1}$ & 842 & $\mathbf{1 8 3}$ & 723 \\
Average & $\mathbf{2 1 4 . 0}$ & 811.0 & $\mathbf{1 9 6 . 7}$ & 813.8 & $\mathbf{2 7 3 . 2}$ & 779.2 & $\mathbf{2 1 1 . 0}$ & 772.7 & $\mathbf{1 7 5 . 7}$ & 717.3 \\
\hline
\end{tabular}

Table 4. Detects average run time in seconds on 300 image data and various noise levels from 5 to 90 comparison of competitive algorithms

\begin{tabular}{cccccc}
\hline & $\sigma=5$ & $\sigma=25$ & $\sigma=45$ & $\sigma=75$ & $\sigma=90$ \\
\hline SNLM [12] & 83.53 & 84.14 & 76.42 & 77.89 & 84.15 \\
BM3D [5] & 4.92 & 6.45 & 7.36 & 6.82 & 6.67 \\
BM3D-SAPCA [13] & 226.43 & 179.13 & 193.35 & 202.67 & 184.38 \\
FastNLM [14] & 0.72 & 0.93 & 0.94 & 0.80 & 0.68 \\
WNNM [11] & 293.24 & 284.31 & 296.57 & 256.89 & 293.73 \\
K-SVD [4] & 95.43 & 156.42 & 153.32 & 168.89 & 172.81 \\
FNSCR [15] & 33.93 & 32.41 & 36.82 & 39.6 & 47.89 \\
Proposed method & 19.42 & 27.13 & 29.42 & 28.54 & 37.02 \\
\hline
\end{tabular}

Table 5. Displays the average PSNR contrast on 300-image data collection at various noise levels 5 to 90 between the proposals and the other state of the art approaches. The bold values reflect the highest PSNR values

\begin{tabular}{|c|c|c|c|c|c|}
\hline & $\sigma=5$ & $\sigma=25$ & $\sigma=45$ & $\sigma=75$ & $\sigma=90$ \\
\hline BM3D [5] & 36.34 & 27.63 & 25.02 & 23.46 & 21.55 \\
\hline LSSC [9] & 36.35 & 28.03 & 26.34 & 26.04 & 24.14 \\
\hline WNNM [11] & 36.41 & 31.52 & 27.03 & 26.93 & 24.25 \\
\hline EPID [10] & 36.58 & 31.56 & 27.52 & 26.56 & 23.94 \\
\hline SNLM [12] & 29.53 & 27.82 & 25.26 & 22.94 & 21.46 \\
\hline BM3D-SAPCA [13] & 36.83 & 28.41 & 26.35 & 24.63 & 22.64 \\
\hline FastNLM [14] & 30.63 & 27.47 & 26.08 & 23.21 & 21.56 \\
\hline FNSCR [15] & 33.09 & 30.79 & 28.83 & 27.53 & 24.09 \\
\hline proposed & 33.56 & 31.32 & 29.58 & 27.83 & 26.36 \\
\hline
\end{tabular}

as affected by the Gaussian noise (GN), noise levels $\sigma=5,25,45,75$, and 90 . The experimental results of this paper reveal that the proposed method has a shorter deployment time. On the other hand, the BM3D and FastNLM methods are quicker than the standard, although the overall suggested output is better than any of the other algorithms. The proposed method maintains certain cooperation between the output of denoised images and the processing period. Natural images are applied to the proposed method. Table 5 shows the average PSNR output on the 300 test images using 08 separate state-of-the-art methods 
under different noise levels. Table 4 displays the average running time of the closing methodologies on the 300 test images. The FastNLM is by far the most efficient method in terms of execution time. It is also efficient in the reduction of worst noise as shown in Table 4. The method proposed offers the highest reduction of noise which is the worst-case during the WNNM processing rate. The methods find cooperation between run time and visual efficiency. The performance of BM3D is simply referred to as a benchmark algorithm because of its capacity to use block similarity and lightweight representation.

Since BM3D utilizes well-defined square blocks with well-defined scale and square shape throughout the image, it results in restricted edges, particularly on strongly contrasting edges. The BM3D-SAPCA variation implements an integrated shape strategy for the community, which enhances visual performance and reduces the implementation time. According to BM3D-SAPCA, the proposed method decreases implementation time due to the usage of the prelearning dictionary that allows time to measure the learning dictionary. The proposed method is slightly better than the BM3D-SAPCA visual output as the proposed method utilizes SRs and meta-heuristic algorithms (DMS with PSO algorithm).

Due to the use of blocks with fixed square form and defined image scale, BM3D performance with edges is limited, particularly with strong contrasting edges. The latest edition, which utilizes an integrated neighbourhood methodology from BM3D-SAPCA, enhances efficiency in visual quality and greatly decreases run time. The proposed method is higher in terms of efficiency and run time compared to BM3DSAPCA and K-SVD. The run time of the proposed method is both higher and quicker than the FNCSR method. The proposed method effectively improves the visual output at a suitable time.

In short, this paper has improved two important aspects. The first is the pre-learned dictionary, resulting in reducing run time compared to the original algorithm. The pre-learned dictionary is recommended for the proposed method, while it requires more than $82 \mathrm{~s}$ for image processing to operate. The computational complexity of the proposed method is less than the local edition of the proposed algorithm. The second aspect is DMS, which has enhanced image searching at the best atom and the effectiveness of the denoised images.

\section{Conclusion}

This paper introduces a dictionary, which was pre-learned. As a result, high-quality images were achieved after using this dictionary. DMS has been used to increase the quality of the image. Such improvements lead to substantial benefits of up to $92 \%$ in computational capacity without losing too much image quality as compared to the initial method. These enhancements have major benefits in their efficiency (PSNR) and implementation time. Detailed research analysis has shown that the performance of the proposed technique in visual quality assessment is lower than other competitive methods as well as execution time measurement for various images and noise rates. The final output testing for an initial algorithm is not sensitive to the elements of the image used for the development of the dictionary. To improve the initial method, the prelearned dictionary method was implemented. The testing has proved that the efficiency of the method is higher than the initial algorithms and the version state.

\section{Conflicts of Interest}

The authors declare no conflict of interest

\section{Author Contributions}

Methodology, FPSO-MP; software, Matlab; validation, 2016R; formal analysis; data duration, BSD500;

\section{References}

[1] E. K. Elsayed, D. R. Salem, and M. Aly, "A Fast Quantum Particle Swarm Optimization Algorithm for Image Denoising Problem", International Journal of Intelligent Engineering and Systems, Vol.13, No.1, pp. 98-112, 2020.

[2] E. K. Elsayed and M. Aly, "Hybrid between Ontology and Quantum Particle Swarm Optimization for Segmenting Noisy Plant Disease Image", International Journal of Intelligent Engineering and Systems, Vol. 12, No. 5, pp. 299-311, 2019.

[3] E. K. Elsayed, D. R. Salem, and M. Aly, "A fast image denoising based on TLS model and sparse representation", International Journal of imaging and robotics, Vol. 19, No. 2, pp. 68-86, 2019.

[4] R. M. Farouk, M. Elsayed, and M. Aly, "Medical image denoising based on log-Gabor wavelet dictionary and K-SVD algorithm", International Journal of Computer Applications, Vol. 141, No. 1, pp. 27-32, 2016.

[5] Z. Zheng, W. Chen, Y. Zhang, S. Chen, and D. Liu, "Denoising the space-borne high-spectralresolution lidar signal with block-matching and 3D filtering", Applied Optics, Vol. 59, No. 9, pp. 2820-2828, 2020. 
[6] Y. Ding, K. Zhou, and W. Bi, "Feature selection based on hybridization of genetic algorithm and competitive swarm optimizer", Soft Computing, pp. 1-10, 2020.

[7] P. Arbelaez, M. Maire, C. Fowlkes, and J. Malik, "Contour detection and hierarchical image segmentation", IEEE Trans. Pattern Anal. Mach. Intell., Vol. 33, No. 5, pp. 898-916, 2011.

[8] Z. Yang and A. Wu, "A non-revisiting quantumbehaved particle swarm optimization based multilevel thresholding for image segmentation", Neural Computing and Applications., pp. 1-21, 2019.

[9] J. Mairal, F. Bach, J. Ponce, G. Sapiro, and A. Zisserman, "Non-local sparse models for image restoration", ICCV., Vol. 29, 2009.

[10] F. Guo, Z. Caiming, and Z. Mingli, "Edge preserving image denoising", IET Image Processing, Vol. 12, No. 8, pp. 1394-1401, 2018.

[11] X. Liu, X. Jing, G. Tang, F. Wu, and Q. Ge, "Image denoising using weighted nuclear norm minimization with multiple strategies", Signal Processing, Vol. 135, pp. 239-252, 2017.

[12] X. Li, H. He, R. Wang, and J. Cheng, "Superpixel-guided nonlocal means for image denoising and super-resolution", Signal Processing, Vol. 124, pp. 173-183, 2016.

[13] V. Katkovnik, A. Foi, K. Egiazarian, and J. Astola, "From local kernel to nonlocal multiplemodel image denoising", International Journal of Computer Vision, Vol. 86, No. 1, pp. $1,2010$.

[14] O. Seynnes and N. Cronin, "Simple Muscle Architecture Analysis (SMA): an ImageJ macro tool to automate measurements in B-mode ultrasound scans", arXiv Preprint arXiv, Vol. 1905, No. 09490, 2019.

[15] X. Shaoping, X. Yang, and S. Jiang, "A fast nonlocally centralized sparse representation algorithm for image denoising", Signal Processing, Vol. 131, pp. 99-112, 2017. 